\title{
ZUR MASSENSPEKTROMETRIE EINIGER DERIVATE VON 4-AZIDO-4-DESOXYPENTOSEN
}

V. Kovã̌tik, C. Pectar, Š. Bauer

Chemisches Institut der Slowakischen Akademie der Wissenschaften, Bratislava 9 (Tschechoslovakei)

UND H. F. GRÜTZMACHER

Institut für Organische Chemie und Biochemie der Universität, Hamburg 13 (Deutschland).

(Eingegangen am 9. März 1971; akzeptiert am 20. April 1971)

\section{ABSTRACT}

The mass spectra of methyl 4-azido-4-deoxypentosides were investigated by high-resolution mass spectrometry and determination of metastable transitions. In addition to compounds having free hydroxyl groups at position 2 and 3, 2,3-di-Omethylsulfonyl, 3-O-acetyl-2-O-meihylsulfonyl, 2,3-di- $O$-benzoyl, 3-O-methyl, and 2,3-anhydro derivatives have been studied. Schemes for the fragmentation of these substances are given.

\section{ZUSAMMENFASSUNG}

Das massenspektrometrische Verhalten von Methyl-4-azido-4-desoxypentosiden wurde mit Hilfe der hochauflösenden Massenspektrometrie und durch Bestimmung der metastabilen Übergānge untersucht. Neben Verbindungen mit freien Hydroxygruppen in 2- und 3-Stellung wurden die 2,3-Di- $O$-methylsulfonyl-, 3-O-Acetyl-2-Omethylsulfonyl-, 2,3-Di- $O$-benzoyl- und 3-O-Methyl-Derivate sowie die 2,3-AnhydroVerbindungen analysiert. Für die Fragmentierungen dieser Verbindungen werden Zerfallsschemata angegeben.

EINFÜHRRUNG

Azidozucker sind wichtige $Z$ wischenprodukte zur Synthese von Aminozuckern. Die Einführung der Azidogruppe gelingt in der Regel durch nukleophile Substitution der entsprechenden Sulfonate ${ }^{1-7}$ oder durch Öffnung eines Epoxidringes ${ }^{8,9} \mathrm{mit}$ Azid-Anionen. Das Verhalten von Azidozuckern beim Elektronenbeschuss im Massenspektrometer wurde bisher noch nicht untersucht. Um die Massenspektrometrie bei der Charakterisierung dieser wichtigen Substanzklasse einsetzen zu können, haben wir daher die massenspektrometrische Fragmentierung von Methylsulfonyl-, Acetyl-, Methyl- und Benzoyl-Derivaten von Methyl-4-azido-4-desoxypentosiden und Methyl2,3-anhydropentosiden studiert. Um einen eventuellen Einfluß der Molekülgeometrie 
auf die Massenspektren zu erkennen, wurden Derivate der L-Arabinose $(1,2,5,6.7)$, D-Xylose $(3,4,8,12,14)$, D-Arabinose (11), L-Xylose $(9,10,13)$ sowie 2,3-AnhydroD-ribose (15) und 2,3-Anhydro-L-ribose (16) in die Untersuchung einbezogen.
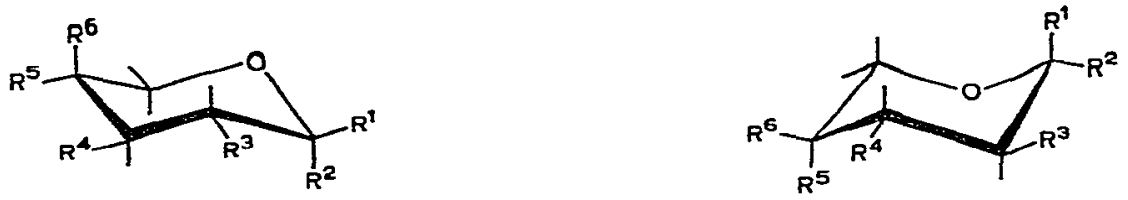

\begin{tabular}{|c|c|c|c|c|c|c|c|c|c|c|c|c|}
\hline & $\mathbf{R}^{1}$ & $\mathbf{R}^{2}$ & $\mathbf{R}^{\mathbf{3}}$ & $\mathbf{R}^{4}$ & $\mathbf{R}^{5}$ & $R^{6}$ & & $R^{1}$ & $\mathbf{R}^{2}$ & $\mathbf{R}^{\mathbf{3}}$ & $\mathbf{R}^{4}$ & $\mathbf{R}^{3}$ \\
\hline 1 & OMe & $\mathbf{H}$ & OMs & OMs & H & $\mathbf{N}_{\mathbf{3}}$ & 9 & OMe & H & OH & $\mathrm{OH}$ & $\mathbf{H}$ \\
\hline 2 & H & OMe & OMs & OMs & $\mathbf{H}$ & $\mathbf{N}_{\mathbf{3}}$ & 10 & & OMe & OH & $\mathrm{OH}$ & $\mathbf{H}$ \\
\hline 3 & H & OMe & OMs & OMs & $\mathbf{N}_{3}$ & $\mathbf{H}$ & 11 & H & OMe & OH & $\mathrm{OH}$ & $\mathbf{N}_{\mathbf{3}}$ \\
\hline & $\mathrm{OMe}$ & & OMs & OMs & $\mathrm{N}_{3}$ & $\mathbf{H}$ & 13 & OMc & $\mathbf{H}$ & OH & OMe & $\mathbf{H}$ \\
\hline 5 & H & oMe & OMs & OAC & $\mathrm{H}$ & $\mathbf{N}_{3}$ & & & & & & \\
\hline 6 & OMe & & OH & $\mathbf{O H}$ & $\mathbf{H}$ & $\mathrm{N}_{3}$ & & & & & & \\
\hline & & OMe & OH & $\mathrm{OH}$ & H & $\mathbf{N}_{3}$ & & & & & & \\
\hline 8 & H & OMe & OH & $\mathrm{OH}$ & $\mathbf{N}_{3}$ & $\mathbf{H}$ & & & & & & \\
\hline 12 & H & $\mathrm{OMe}$ & OH & $\mathrm{OMe}$ & $\mathbf{N}_{3}$ & H & & & & & & \\
\hline 14 & H & OMe & $\mathrm{OBz}$ & $\mathrm{OBz}$ & $N_{3}$ & H & & & & & & \\
\hline
\end{tabular}

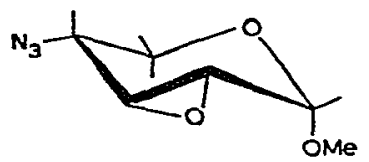

15

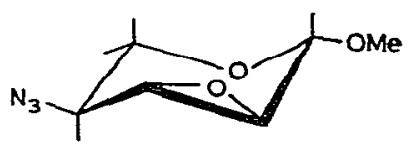

16

EXPERIMENTELLER TEIL

Die Massenspektren wurden bei einer Elektronenenergie von $70 \mathrm{eV}$ bei direktem Einlass in die Ionenquelle mit dem Gerät MCh 1360 (UdSSR) aufgenommen. Die Verbindungen 1-4, 6, 7, 9, 10, 15 und 16 wurden zum Vergleich auch mit einem Massenspektrometer SM-1B, VARIAN MAT, bei direktem Substanzeinlass und $70 \mathrm{eV}$ Elektronenenergie untersucht. Mit letzterem Gerät wurden genaue Massenbestimmungen mit einem Fehler von 10 p.p.m. durchgeführt. Die so erhaltenen Summenformeln der verschiedenen Ionen sind in den Schemata und im Text in Klammern angeführt. Die für die Aufstellung der Zerfallsreihen wichtigen metastabilen Übergänge wurden Massenspektren entnommen, die mit dem einfachfokussierenden MCh-1306-Massenspektrometer erhalten wurden. Diese und zusātzliche metastabile Übergänge wurden im SM-1B-Massenspektrometer mit Hilfe der Defokussierungstechnik gleichfalls nachgewiesen. .

ERGEBNISSE UND DISKUSSION

Die Massenspektren von Methyl-2,3,4-tri- $O$-methylsulfonylpentopyranosiden und Methyl-3-O-acetyl-2,4-di- $O$-methylsulfonylpentopyranosiden wurden bereits früher untersucht ${ }^{11}$. Fragmentierungen vom ähnlichen Typ werden auch in den 
Massenspektren der 2,3-Di- $O$-methylsulfonylderivate 1-4 und des 3-O-Acetyl-2-Omethylsulfonylderivats 5 der Methyl-4-azido-4-desoxypentoside beobachtet. So wird der Basispeak in den Massenspektren von 1-4 bei m/e 79 von $\mathrm{CH}_{3} \mathrm{SO}_{2}^{+}$-Ionen, der im Spektrum von 5 bei $m / e 43$ von $\mathrm{CH}_{3} \mathrm{CO}^{+}$-Ionen gegeben. Die Azidogruppe an C-4 bedingt jedoch wichtige Unterschiede beim Zerfall der Verbindungen 1-5 im Massenspektrometer, die im Schema 1 am Beispiel des Methyl-4-azido-4-desoxy-2,3di- $O$-methylsulfonyl- $\alpha$-D-xylopyranosids (3) (Massenspektrum Abb. 1) angegeben
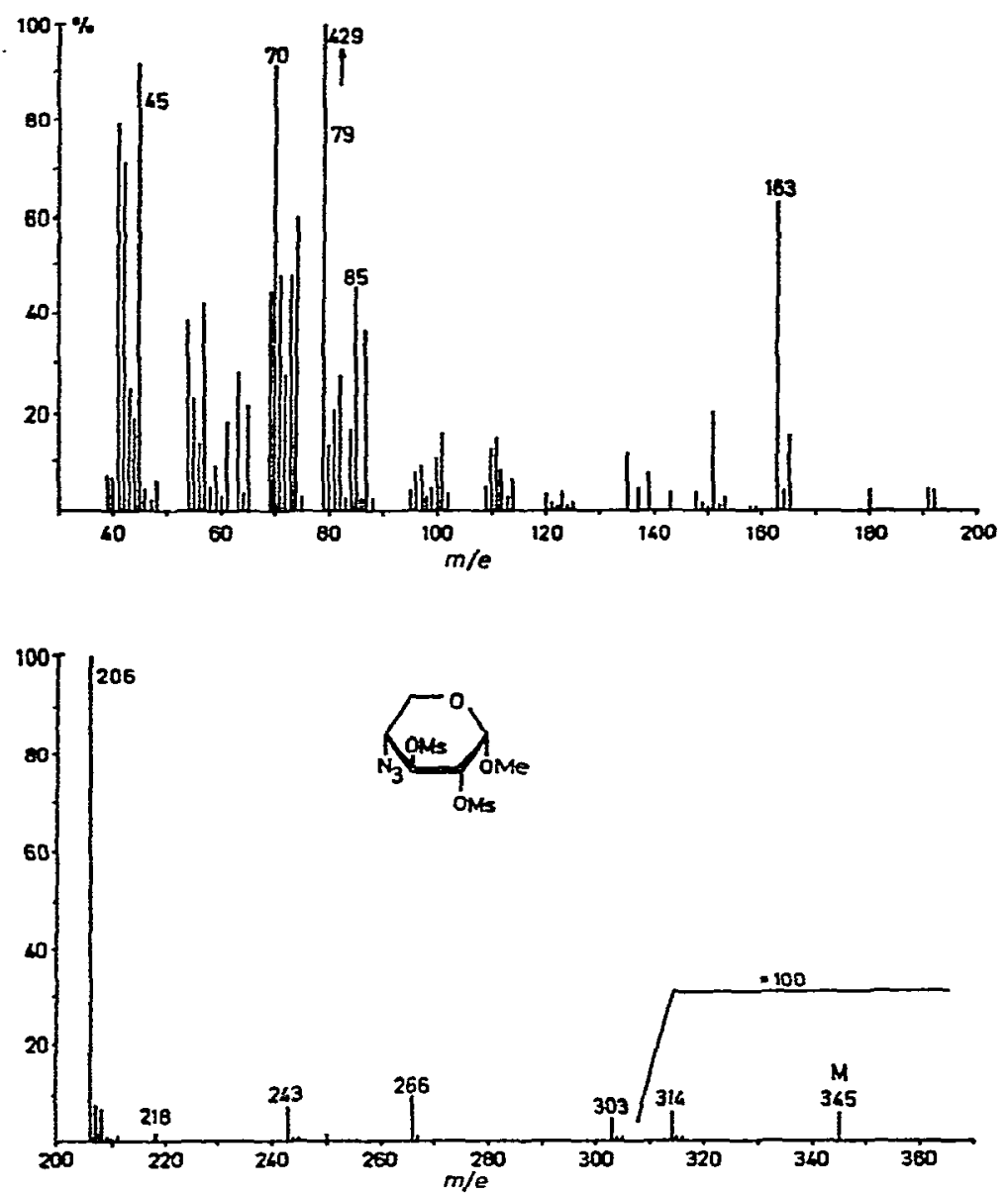

Abb. 1. Massenspektrum des Methyl-4-azido-4-desoxy-2,3-di- $O$-methylsulfonyl- $\alpha$-D-xylopyranosids.

sind. Die in Klammern aufgeführten Massenzahlen beziehen sich auf die Fragmentierung des Methyl-3- $O$-acetyl-4-azido-4-desoxy-2- $O$-methylsulfonyl- $\beta$-L-arabopyranosids (5), dessen Massenspektrum in Abb. 2 wiedergegeben ist.

Die für die Molekül-Ionen von Methylglykosiden typische Abspaltung der glykosidischen Methoxygruppe, gefolgt von der Eliminierung weiterer Substituenten 

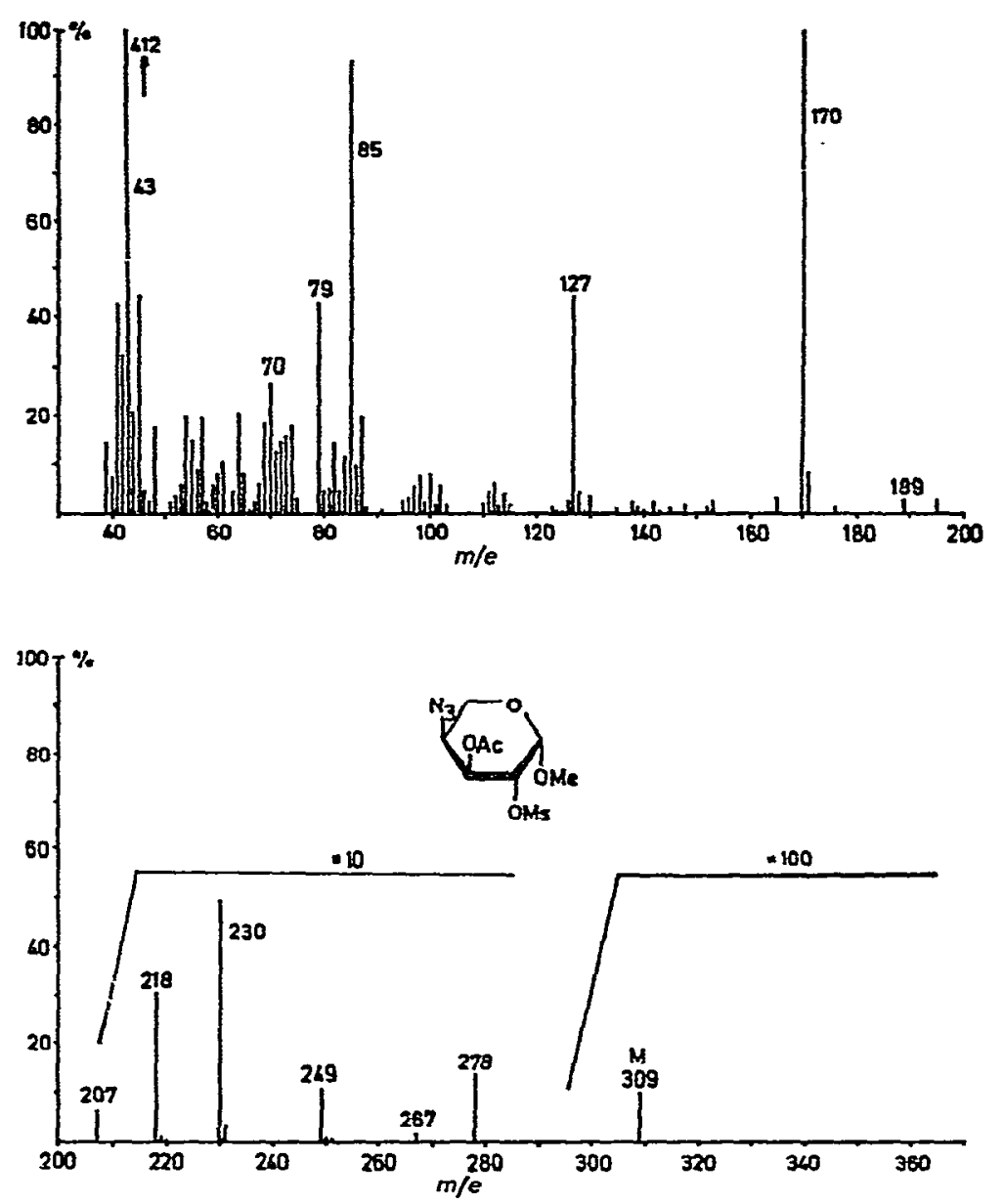

Abb. 2. Massenspektrum des Methyl-3- $O$-acetyl-4-azido-4-desoxy-2- $O$-methylsulfonyl- $\beta$-L-arabopyranosids.

unter Ausbildung konjugiert ungesättigter Oxonium-Ionen, besitzt in den Massenspektren von 1-5 nur geringe Bedeutung. Der bevorzugte Abbauweg der MolekülIonen beginnt mit der Abspaltung eines $\mathrm{CH}_{3} \mathrm{SO}_{2}$-Radikals, gefolgt von der Eliminierung von $\mathrm{HCO}_{2} \mathrm{CH}_{3}$ (aus dem Ringsauerstoffatom und der C-1-Gruppe) zu Ionen der Masse 206 (170), deren Peak den oberen Massenbereich des Spektrums beherrscht. Der weitere Zerfall erfolgt durch Abspaltung von $\mathrm{HN}_{3}$, während eine Abspaltung von $\mathrm{CH}_{3} \mathrm{SO}_{3} \mathrm{H}$ bzw. $\mathrm{CH}_{3} \mathrm{CO}_{2} \mathrm{H}$ nur mit sehr geringer Intensität eintritt. Die Mesyl- bzw. Acetyl-gruppe an C-3 beteiligt sich erst nach der $\mathrm{HN}_{3}$-Eliminierung durch Abspaltung von $\mathrm{CH}_{2} \mathrm{SO}_{2}$ oder $\mathrm{CH}_{2} \mathrm{CO}$ an der Fragmentierung.

Bei einem Vergleich der relativen Intensitäten der Peaks m/e 206 und m/e 163 in den Massenspektren der Stereoisomeren 1-4 zeigt sich, dass die Abspaltung von $\mathrm{HN}_{3}$ aus den Ionen m/e 206 bei 1 und 2 mit ursprünglich axialer Azidogruppe im intakten Molekül 1,8-2 mal leichter erfolgt als bei den Isomeren 3 und 4 mit ursprüng- 


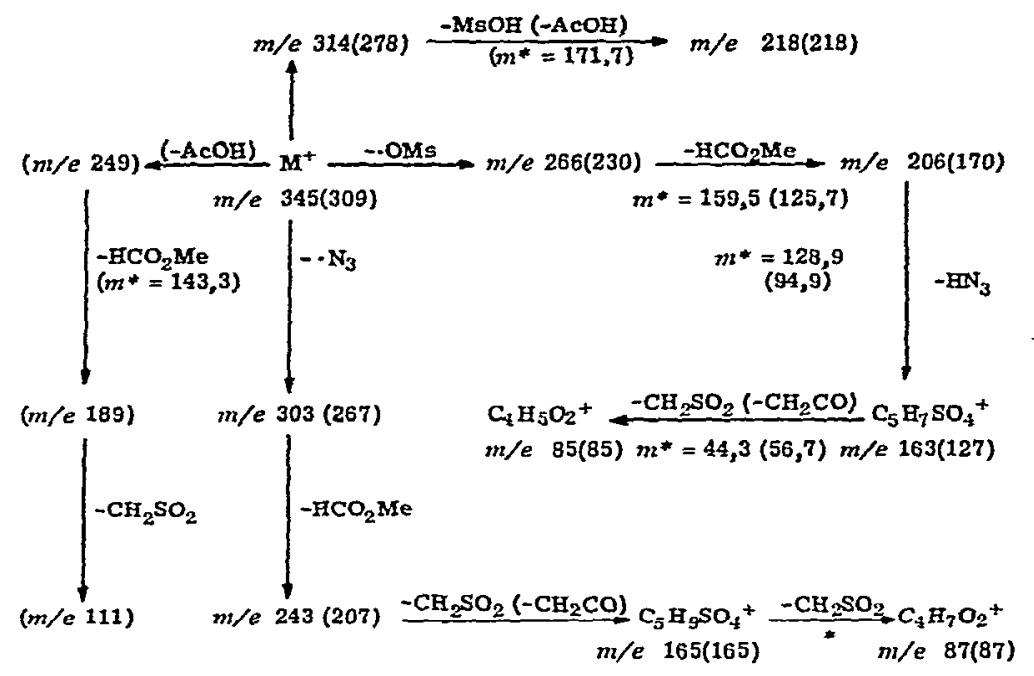

Schema 1

lich ãquatorial stãndigen Azid-Substituenten (Tabelle I). Dieser Effekt wurde übereinstimmend sowohl bei den Untersuchungen mit dem Massenspektrometer $\mathrm{MCh}$ 1306 als auch mit dem Massenspektrometer SM-1B gemessen und ist daher wenig abhāngig von den Aufnahmebedingungen. Er kann deswegen gut zur Bestimmung der Konfiguration der Azidogruppe in den Methansulfonylestern der 4-Azido-4desoxypentoside herangezogen werden.

TABELLE I

VERGLEICH DER MASSENSPEKTREN VON VERBINDUTNGEN $1-4$

\begin{tabular}{|c|c|c|c|}
\hline \multirow[t]{2}{*}{ Verbindung } & \multirow[t]{2}{*}{$\begin{array}{l}\text { Orientierung der Azid-Gruppe } \\
\text { am C-4 }\end{array}$} & \multicolumn{2}{|c|}{$\frac{[\mathrm{m} / \mathrm{e} 163]}{[\mathrm{m} / \mathrm{e} 206]} \times 100$} \\
\hline & & $M C h 1306$ & $S M-1$ \\
\hline 1 & Axial & 115 & 109 \\
\hline 2 & Axial & 115 & 107 \\
\hline 3 & Äquatorial & 64 & 56 \\
\hline 4 & Äquatorial & 82 & 61 \\
\hline
\end{tabular}

Außer durch Verlust von $\mathrm{CH}_{3} \mathrm{SO}_{2}^{-}$kann der Abbau der Molekül-Ionen von 1-5 auch durch $A$ bspaltung von $\mathrm{N}_{3}$ eingeleitet werden. gefolgt von einer Eliminierung von $\mathrm{HCO}_{2} \mathrm{CH}_{3}$ und $\mathrm{CH}_{2} \mathrm{SO}_{2}$ oder $\mathrm{CH}_{2} \mathrm{CO}$, so daß Ionen der Massen 303 (267), 243 (207), 165 und 87 entstehen. Wie die hochaufgelösten Massenspektren zeigen, ist der Peak m/e 165 ein Dublett der Ionen $\mathrm{C}_{5} \mathrm{H}_{9}^{32} \mathrm{SO}_{4}^{+}$und $\mathrm{C}_{5} \mathrm{H}_{7}^{34} \mathrm{SO}_{4}^{+}$im Intensitätsverhältnis 5:1.

In den Massenspektren von permethylierten und peracetylierten Pentosen, Hexosen ${ }^{12}$ und Uronsäuren ${ }^{13}$ wird einer đer größten Peaks von Ionen geliefert, die 
die C-Atome 2-4 und die Substituenten der C-Atome 2 und 4 enthalten. Diese Ionen werden auch noch bei der Fragmentierung der Methansulfonylester von Pentosen ${ }^{11}$ beobachtet. In den Massenspektren von 1-5 müßten sie die Struktur $\mathrm{N}_{3}-\mathrm{CH}=\mathrm{CH}-$ $\mathrm{CH}=\stackrel{\mathrm{OSOO}}{2} \mathrm{CH}_{3}$ und die Masse 176 besitzen. Ein größerer Peak bei dieser Massenzahl wird aber nicht gefunden. Stickstoffhaltige Ionen treten im unteren Massenbereich bei $m / e 98\left(\mathrm{C}_{3} \mathrm{H}_{4} \mathrm{~N}_{3} \mathrm{O}\right)$, m/e $72\left(\mathrm{C}_{3} \mathrm{H}_{6} \mathrm{NO}\right)$, m/e $71\left(\mathrm{C}_{3} \mathrm{H}_{5} \mathrm{NO}\right)$, m/e $70\left(\mathrm{C}_{3} \mathrm{H}_{4} \mathrm{NO}\right)$ sowie $m / e 42\left(\mathrm{C}_{2} \mathrm{H}_{4} \mathrm{~N}\right.$ neben $\left.\mathrm{C}_{2} \mathrm{H}_{2} \mathrm{O}\right)$ auf. Diese Ionen entstehen jedoch durch einen so weitgehenden Abbau der Molekül-Ionen, dass ihre Bildungsreaktionen schwierig zu ermitteln sind.

Der Zerfall dez Molekül-Ionen der Methyl-4-azido-4-desoxypentopyranoside 6-11 wird am Beispiel des Massenspektrums des Methyl-4-azido-4-desoxy- $\beta$ - $L-$ xylopyranosids (10) (Abb. 3) im Schema 2 formuliert.

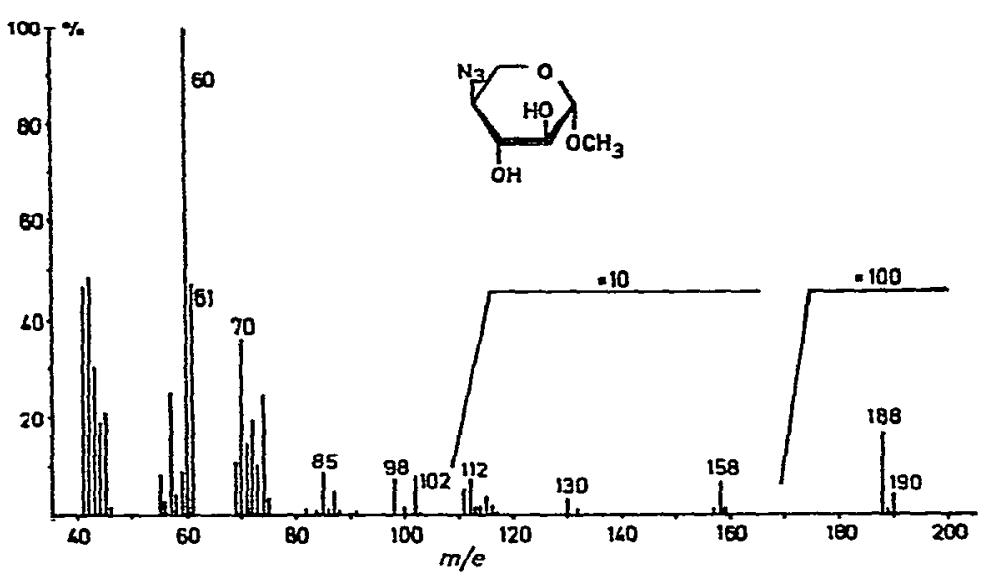

Abb. 3. Massenspektrum des Methyl-4-azido-4-desoxy- $\beta$-L-xylopyranosids.

Wie in den Massenspektren anderer partiell methylierter Zucker sind auch bei den Spektren dieser Verbindungen die Peaks im oberen Massenbereich klein, jedoch lassen sich $M+1$ - und $M-1$-Ionen bei $m / e 190$ und $m / e 188$ in allen Spektren nachweisen. Die Fragmentierung von 6-11 besitzt Ähnlichkeit mit dem Zerfall partiell methylierter Methylpentoside im Massenspektrometer. So erfolgt wie bei anderen Pyranosiden bevorzugt die Bildung von Ionen aus den Ring-C-Atomen C-2 una $\mathrm{C}-3^{12}$ mit der Struktur $\mathrm{HO}-\mathrm{CH}=\mathrm{CH}-\mathrm{OH}^{+}$und der Masse 60 . In geringerer Menge werden durch eine ähnliche cyclische Elektronenverschiebung im Pyranosering der Molekül-Ionen auch $\mathrm{CH}_{3} \mathrm{O}-\mathrm{CH}=\mathrm{CH}-\mathrm{OH}^{+}$-Ionen mit $m / e 74$ gebildet. Die Massenspektren der Methyläther von Zuckern zeichnen sich durch eine Umlagerungsreaktion aus, bei der durch Wanderung des Substituenten von C-3 an C-1 Ionen der Srruktur $\mathrm{CH}_{3} \mathrm{O}-\mathrm{CH}-\mathrm{+}-\mathrm{OR}$ entstehen. Auf diese Weise lässt sich in den Massenspektren von 6-11 die Bildung von $\mathrm{CH}_{3} \mathrm{O}-\stackrel{+}{\mathrm{C}} \mathrm{H}-\mathrm{OH}$ mit der Masse 61 erklāren. Auch die Ionen $\mathrm{N}_{3}-\mathrm{CH}=\mathrm{CH}-\mathrm{CH}=\grave{\mathrm{O}} \mathrm{H}$ mit $m / e 98$ entstehen durch eine für Saccharide typische Carbohyd. Res., 19 (1971) 169-177 
Fragmentierungsreaktion. Ihre geringe Intensität zeigt aber an, dass die positive Ladung in diesen Ionen nicht gut stabilisiert werden kann. Der weitere Zerfall der Ionen $m / e 98$ erfolgt in zwei Reaktionsschritten durch Eliminierung von $\mathrm{N}_{2}$ und $\mathrm{CO}$ zu Ionen der Massen 70 und 42.

Wie bei zahlreichen anderen Methylglykosiden wird aus den Molekül-Ionen von 6-11 die glykosidische $\mathrm{CH}_{3} \mathrm{O}$-Gruppe leicht abgespalten. Die so gebildeten Ionen der Masse 158 zerfallen in einer für 4-Azido-4-desoxy-zucker charakteristischen Reaktion durch Abspaltung von $\mathrm{N}_{2}$ leicht weiter. Der Abbau läßt sich durch metastabile Ubergänge gut verfolgen (Schema 2). Es muß jedoch damit gerechnet werden, dass mit der $\mathrm{N}_{2}$-Eliminierung eine weitgehende Umlagerung einhergeht, so daß Strukturen der entstehenden Ionen der Massen 130, 112, 102 und 85 unbekannt sind.

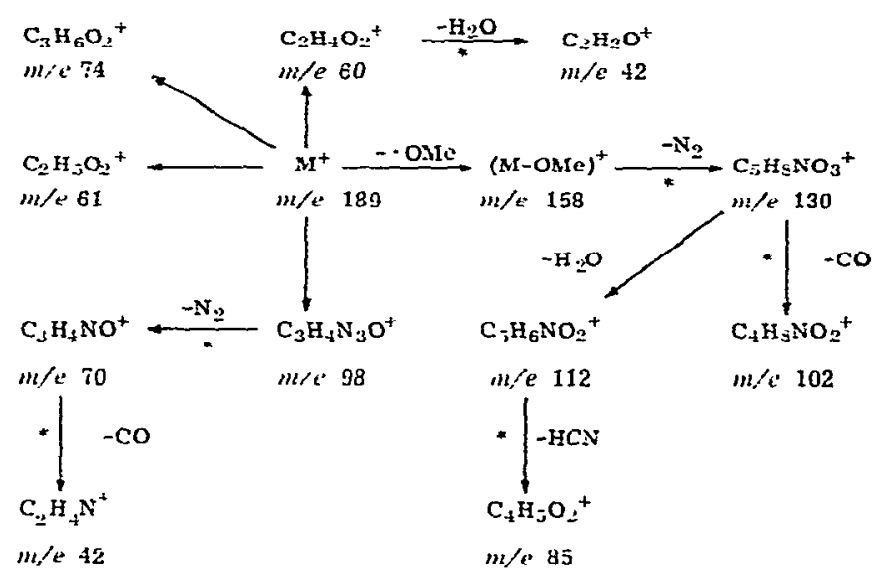

Schema 2

Die Fragmentierung der Methyl-4-azido-4-desoxy-3-O-methylpentopyranoside 12 und 13 (Abb. 4) erfolgt auf dem gleichen Wege wie bei 6-11. Die Methoxygruppe an $\mathrm{C}-3$ bewirkt jedoch eine Masseverschiebung der Ionen m/e 60 und 61 nach $m / e$ 74 und 75 und zugleich eine Ladungsstabilisierung in diesen Ionen. Die Peaks bei $m / e 74$ und 75 dominieren daher in den Massenspektren, die der übrigen Ionen werden in ihren Intensitäten herabgesetzt. Die Massenspektren der stereoisomeren Verbindungen 6-11 sowie 12 und 13 zeigen nur geringfügige Unterschiede, die im Rahmen der experimentellen Fehler liegen. Eine massenspektrometrische Bestimmung der Stereochemie dieser Derivate ist daher nicht möglich.

Methyl-4-azido-2,3-di- $O$-benzoyl-4-desoxy- $\alpha$-D-xylopyranoside (14) wird im Massenspektrometer durch entsprechende Reaktionen abgebaut wie die Verbindungen 1-13. Die Untersuchung der Benzoylderivate der Azidozucker bietet im Vergleich zu den übrigen Derivaten keine Vorteile, da die Bildung von Ionen der Masse 105 aus den Benzoylgruppen ausserordentlich bevorzugt wird. 


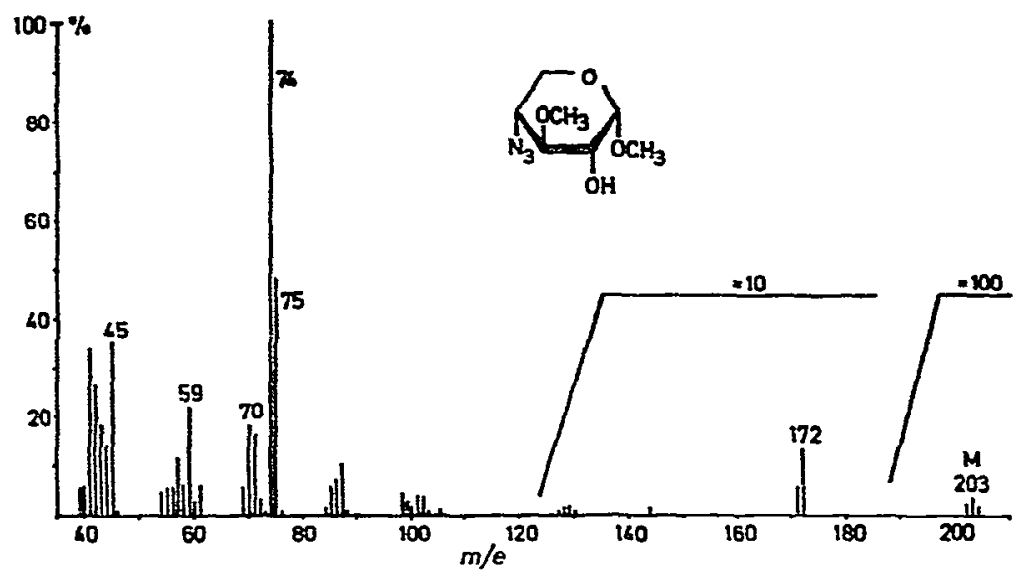

Abb. 4. Massenspektrum des Methyl-4-azido-4-desoxy-3-O-methyl-az-D-xylopyranosids.

Der Zerfall der Molekül-Ionen der Methyl-2,3-anhydro-4-azido-4-desoxy pentopyranoside 15 und 16 ist im Schema 3 wiedergegeben (Massenspektrum siehe Abb. 5). Auch bei diesen Verbindungen beginnt der Zerfall entweder durch Abspaltung der glykosidischen Methoxygruppe oder durch den Verlust eines $\mathrm{N}_{3}$-Radikals.

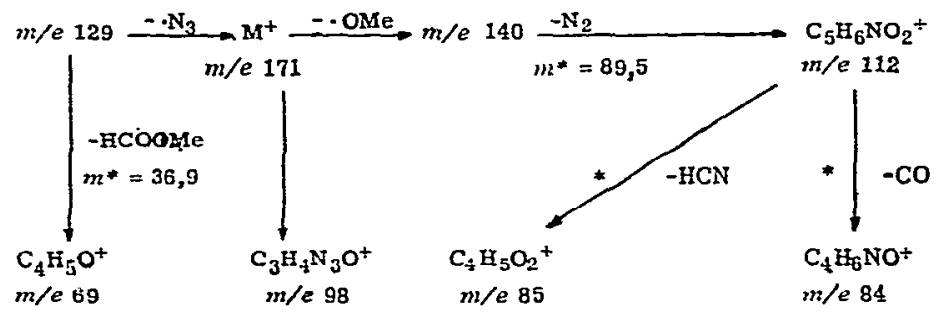

Schema 3

Letztere Fragmentierung führt zu Ionen $m / e$ 129, aus denen ein Molekül $\mathrm{HCO}_{2} \mathrm{CH}_{3}$ zu Ionen der Masse 69 abgespalten wird, die den Basispeak der Spektren liefern. Der Primärspaltung an C-1 unter Verlust der Methoxygruppe zu den Ionen m/e 140 folgt die Abspaltung von $\mathrm{N}_{2}$ zu den Ionen m/e 112. Auch in diesem Fall muss damit gerechnet werden, dass die $\mathrm{N}_{2}$-Abspaltung von einer Skelettumlagerung begleitet wird, so dass die Struktur der Ionen $m / e 112$ unbekannt ist; sie zerfallen weiter durch Abspaltung von $\mathrm{CO}$ zu den Ionen $m / e 84$ oder von $\mathrm{HCN}$ zu den Ionen m/e 85 .

Beim Vergleich der Massenspektren der Stereoisomeren 15 und 16 zeigt sich, dass die glykosidische Methoxygruppe bei axialer Stellung wie in 15 8-9 mal leichter abgelöst wird als bei äquatorialer Stellung wie in 16. Dagegen wird aus den MoleküiIonen von 16 leichter ein $\mathrm{H}$-Atom zu $(\mathrm{M}-\mathrm{H})^{+}$-Ionen mit $m / e 170$ abgespalten (Tabelle II). 


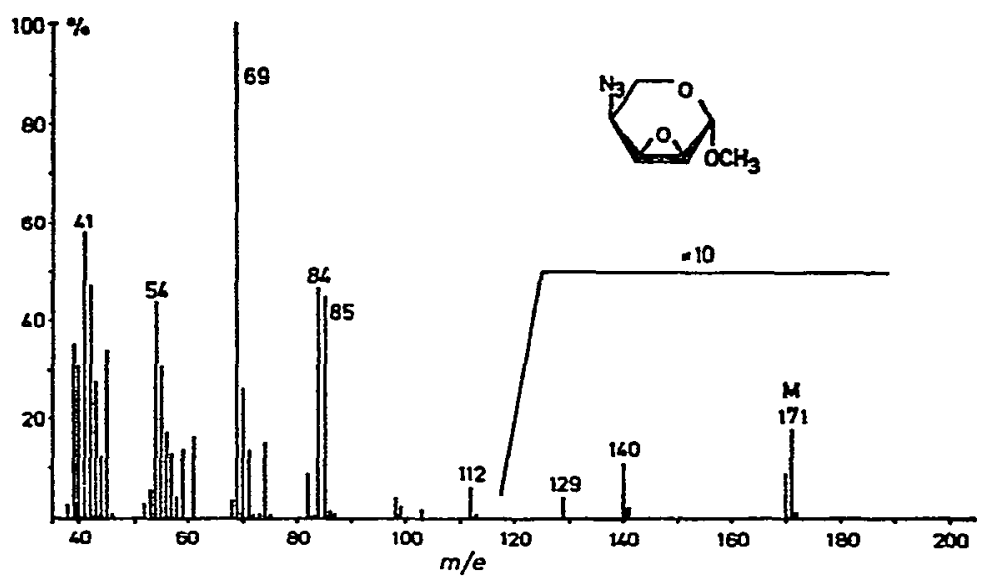

Abb. 5. Massenspektrum des Methyl-2,3-anhydro-4-azido-4-desoxy- $\beta$-I-ribopyranosids.

\section{TABELLE II}

VERGLEICH DER MASSENSPEKTREN YON VERBINDUNGEN 15 UND 16

\begin{tabular}{|c|c|c|c|c|c|}
\hline \multirow[t]{2}{*}{ Verbindung } & \multirow[t]{2}{*}{$\begin{array}{l}\text { Orientierung der Methoxyl- } \\
\text { Gruppe am C-I }\end{array}$} & \multicolumn{2}{|c|}{$\frac{[M-M e O]^{+}}{[M]^{+}} \times 100$} & \multicolumn{2}{|c|}{$\frac{[M-H]^{+}}{[M]^{+}} \times 100$} \\
\hline & & MCh 1306 & $S M-1$ & MCh 1306 & $S M-1$ \\
\hline $\begin{array}{l}15 \\
16\end{array}$ & $\begin{array}{l}\text { Axial } \\
\text { Äquatorial }\end{array}$ & $\begin{array}{c}580 \\
63\end{array}$ & $\begin{array}{c}542 \\
71\end{array}$ & $\begin{array}{r}8 \\
46\end{array}$ & $\begin{array}{l}10 \\
44\end{array}$ \\
\hline
\end{tabular}

DANK

Der Alexander von Humboldt-Stiftung dankt V. Kováčik für ein Forschungsstipendium während des Aufenthaltes am Institut für Organische Chemie und Biochemie der Universität Hamburg.

\section{LITERATUR}

1 E. J. ReIST, R. R. SPENCER, B. R. BAKER und L. Goodman, Chem. Ind. (London), (1962) 1794.

2 J. Hul, L. Hough und A. C. Richardson, Proc. Chem. Soc., (1963) 346.

3 E. J. Reist, R. R. Spencer, D. F. Calkins, B. R. BAKER und L. Goodman, J. Org. Chem., 30 (1965) 2312.

4 E. J. Reist, D. E. Guefrro und L. Goodman, J. Amer. Chem. Soc., 87 (1965) 677.

5 A. J. DICK UND J. K. N. JoNEs, Can. J. Chem., 43 (1965) 977.

6 A. J. Dick und J. K. N. Jones, Can. J. Chem., 44 (1960) 79.

7 C. L. Stevens, P. Blumbergs und D. H. Otterbach, J. Org. Chem., 31 (1966) 2817.

8 R. D. Guthrie und D. Murphy, J. Chem. Soc., (1963) 5288.

9 R. D. GUTHRIE UND D. MuRPHY, J. Chem. Soc., (1965) 3828.

10 T. StICzAY, P. Stros und S. BAuer, Carbohyd. Res., 10 (1969) 469.

11 V. Kovíčik, P. KovÁC und R. L. WhistLeR, Carbohyd. Res., 14 (1970) 133.

12 K. Heyns, H. F. GrützmaCher und H. SCHARMANn, Fortschr. Chem. Forsch., 5 (1966) 448.

13 V. KOYÁČK, S. BAUER, J. Rosík uND P. KovǍ̉, Carbohyd. Res., 8 (1968) 282. 